Revue des patrimoines

\title{
Un dépôt révolutionnaire : des statues de Marly à Bolbec
}

\section{Claire Etienne}

\section{(2) OpenEdition}

\section{Journals}

Édition électronique

URL : http://journals.openedition.org/insitu/1055

DOI : 10.4000/insitu.1055

ISSN : 1630-7305

\section{Éditeur}

Ministère de la culture

Référence électronique

Claire Etienne, « Un dépôt révolutionnaire : des statues de Marly à Bolbec », In Situ [En ligne], 1 | 2001, mis en ligne le 24 avril 2012, consulté le 20 avril 2019. URL : http://journals.openedition.org/ insitu/1055 ; DOI : 10.4000/insitu.1055

Ce document a été généré automatiquement le 20 avril 2019

\section{(c) $($ i) $(9)$}

In Situ Revues des patrimoines est mis à disposition selon les termes de la licence Creative Commons Attribution - Pas d'Utilisation Commerciale - Pas de Modification 4.0 International. 


\title{
Un dépôt révolutionnaire: des statues de Marly à Bolbec
}

\author{
Claire Etienne
}

1 En 1794, la petite ville normande de Bolbec, connue pour son industrie textile est administrée par un maire acquis à la Révolution, dévoué à la cause publique et au développement de sa cité et au bien-être physique et moral de ses habitants, JacquesDominique Ruffin. Son adhésion aux idées nouvelles et à la Révolution française est affichée et il présente lui-même son fils, François-Amable, âgé de vingt et un ans, en septembre 1792, pour signer son engagement dans l'armée de le République à l'appel de la « Patrie en danger ${ }^{1}$.

2 La ville de Bolbec revenait alors de loin. En 1765, alors que la route royale de Rouen au Havre, tracée par les ingénieurs des Ponts et chaussées émules de Charles Trudaine arrive à ses portes ${ }^{2}$, elle est presque entièrement détruite par un incendie ${ }^{3}$. L'élan de solidarité de la province de Normandie, les exemptions et secours royaux devant le désastre permettent une reconstruction rapide dont témoignent les dates portées sur les hôtels et maisons élevées sur les ruines qui s'échelonnent de 1766 à 1780. Les ingénieurs qui auraient voulu profiter de la table rase pour tracer une nouvelle traversée de la ville doivent se contenter d'élargir par des « retranchements » dans les parcelles limitrophes la voie existante, la Grand-rue, devenue après 1790 la rue de la République ${ }^{4}$. Cette voie prend à partir de l'ancienne place du Marché qu'on a conservé et à qui est dévolu le rôle de centre de la cité avec l'implantation de l'hôtel de ville dont la construction commence en $1791^{5}$. Elle aboutit en revanche sur une petite place nouvelle, ménagée depuis l'incendie en dégageant la façade de l'église reconstruite sur des plans de l'architecte parisien Pierre Patte ${ }^{6}$.

3 Le 30 novembre 1793, la municipalité de Bolbec décide de doter la ville d'un réseau urbain des eaux et charge le maire de demander aux administrateurs du département l'autorisation de prendre les bois nécessaires sur les terrains des émigrés ${ }^{7}$. Le 27 avril 1794, est établi le devis de construction d'un château d'eau et d'une conduite en bois de 761 toises de long pour la distribution de l'eau, avec établissement d'une fontaine à quatre 
tuyaux place du Marché, deux autres fontaines dans des quartiers éloignés de la commune. Le château d'eau sera construit sur des fondations en moellon, maçonné et voûté en brique, pavé en grès, avec des marches en pierres de Caumont et une porte en chêne. La "grande fontaine » sera également construite sur des fondations en moellon et pavée de grès, mais ses murs sont en pierre de Caumont avec une base d'ordre toscan et une calotte en forme de voûte et panache en pendentif à l'extérieur's.

4 Le 19 mars 1794, les trois fontaines publiques sont construites, la première près de la maison commune (place du marché), la seconde près de la ci-devant église (la nouvelle place) et la troisième près de la maison du citoyen Louis Caron (rue d'Orteuil) ${ }^{9}$. Le 15 juillet, Jacques-Dominique Ruffin demande à la Convention nationale des figures en marbre pour orner les nouvelles fontaines distribuées sur trois places publiques ${ }^{10}$. Or, le 1er novembre 1793 a eu lieu l'inventaire et l'extraction des objets de peinture et sculptures du château de Marly, susceptibles d'être transportés au Museum national: «avons commencé par une statue moderne de Diane sur un piédestal orné de consoles et de trophées de chasse, cette statue est placée au haut de l'allée des portiques côté de Louveciennes et est désignée sous le numéro 1 et caetera ${ }^{11}$ (voir ci-dessous).

5 Jacques-Dominique Ruffin sollicite donc un groupe du dépôt de Marly et espère que la Commission voudra bien lui accorder « eux autres groupes ou figures à prendre dans les dépôts de Paris ». Le 15 juillet 1794, le sculpteur Du Parquier, membre du Muséum des Arts, donne un certificat attestant que le groupe représentant le Génie des Arts relevé par le Temps et que la statue d'un enfant appuyé sur une gerbe d'eau provenant tous deux de « la destruction des objets de luxe du ci-devant château de Marly , vu leur imperfection marquée pourront être employée de manière avantageuse dans un autre endroit que celui destiné au centre des Arts ». Il est donc favorable à la demande du maire de Bolbec et ne s'oppose pas à leur transfert. Le groupe décrit porte le numéro 35 de l'inventaire des œuvres d'art du parc de Marly: "le groupe du Temps relevé par les Arts (sic) est moderne. Il a son piédestal ».

6 Le 7 décembre 1794, la Commission exécutive de l'Instruction publique délivre aux commissaires de la commune de Bolbec «le groupe représentant le Génie des Arts relevé par le Temps, de six pieds de haut (sans le piédestal), plus un enfant appuyé sur une gerbe d'eau pour être employé, tout en gardant sa qualité de propriété nationale, à la décoration des fontaines publiques de Bolbec ", à charge pour la commune d'acquitter les frais de déplacement ou de transport. On voit que le schéma qui fera florès au XIXe siècle pour les dépôts de l'Etat est déjà établi ${ }^{12}$.

7 Mais réflexion faite, Jacques-Dominique Ruffin se désiste de sa demande de la figure de l'enfant sur la gerbe d'eau, "vu que cette figure ferait mauvais effet sur une place publique, étant disposée pour être adossée à un édifice quelconque ». Sa demande est donc renvoyée au Comité d'Instruction publique, qui délivre au maire de Bolbec «la figure de Diane en marbre qui est dans les magasins de Marly à la place de l'enfant appuyé sur une gerbe d'eau qui lui avait été accordé par arrêté du 16 frimaire $»^{13}$. Le 8 octobre 1795, le conseil municipal, au vu d'un dessin colorié de la fontaine des Arts relevés par le Temps, exécuté par Lange, accepte le dépôt révolutionnaire ${ }^{14}$.

8 Les deux sculptures ainsi attribuées au maire de Bolbec pour l'ornementation de sa ville ont leur pedigree dans les comptes du château de Marly. Le groupe sculpté qui a pris divers titres, dont selon Piganiol de la Force «le Temps qui tire le mérite de l'obscurité et le couronne » a été achetée à Gênes et fournie le 20 décembre 1700 par le marbrier Jérôme Denais : «pour un groupe de marbre représentant le temps qui relève des Arts de sept 
pieds de haut, pour être posé à Marly, 3400 livres ». De même, la statue de Diane figure dans les comptes des bâtiments du roi de 1716 et a été exécutée par le sculpteur Jean Poultier ${ }^{15}$ : «parfait paiement de 3600 livres pour une figure représentant une Nymphe de Diane, qu'il a faite et posée dans le jardin de Marly en 1714, cy 2400 livres ». Son piédestal, inventorié avec la statue en 1793 est l'œuvre du sculpteur Jean Hardy ${ }^{16}$. Commandé en 1716, il est payé 2400 livres en $1717^{17}$.

Pendant presque un siècle, les deux sculptures ornent les fontaines des deux places publiques de Bolbec. Deux photographies anciennes les montrent en situation. La fontaine de la place du Marché est en fait située sur un promontoire bordé de tilleuls, dominant la place en contrebas. C'est un massif de pierre de taille calcaire émergeant d'un bassin circulaire qu'elle alimente de robinets sortant de mufles de lions en bronze sur les quatre côtés. Ce massif est surmonté d'un piédestal cubique mouluré à la base et au sommet avec l'inscription en capitale : MUNIFICENCE NATIONALE ${ }^{18}$. La fontaine devant l'église est déplacée une première fois en 1843 , lorsque la place Diane est définitivement aménagée par l'ouverture d'une nouvelle rue et la destruction d'un îlot. Située d'abord près de l'église elle occupe alors le centre de la nouvelle place. La fontaine est plus petite, c'est un massif maçonné rectangulaire aux angles abattus à un seul tuyau alimentant un bassin de forme rectangulaire à angles arrondis, placé devant. Il est surmonté du piédestal décrit dans l'inventaire de $1793^{19}$.

En 1881, l'hôtel de ville est trop petit pour la ville en expansion et on décide de le reconstruire en prenant sur la fontaine et sur une partie des hallettes, mais la population privée ainsi d'une partie de la place du Marché s'y oppose. La municipalité décide d'approprier en mairie l'hôtel du manufacturier Lemaitre, dont elle vient d'hériter et la place est entièrement dévolue au marché. La place Diane abrite elle aussi une succession de marchés, aux denrées alimentaires, aux poissons puis aux fleurs. Dans ce contexte, les fontaines apparaissent plus une gêne qu'un avantage, et en 1887, "à défaut de pouvoir déplacer les marchés, on déménage les statues », sous la direction du sculpteur Bonnet ${ }^{20}$. Les fontaines sont détruites et les deux sculptures sont placées dans le nouveau jardin public devant le nouvel hôtel de ville, retrouvant ainsi leur vocation d'origine de décor de jardin. 
Figure 1

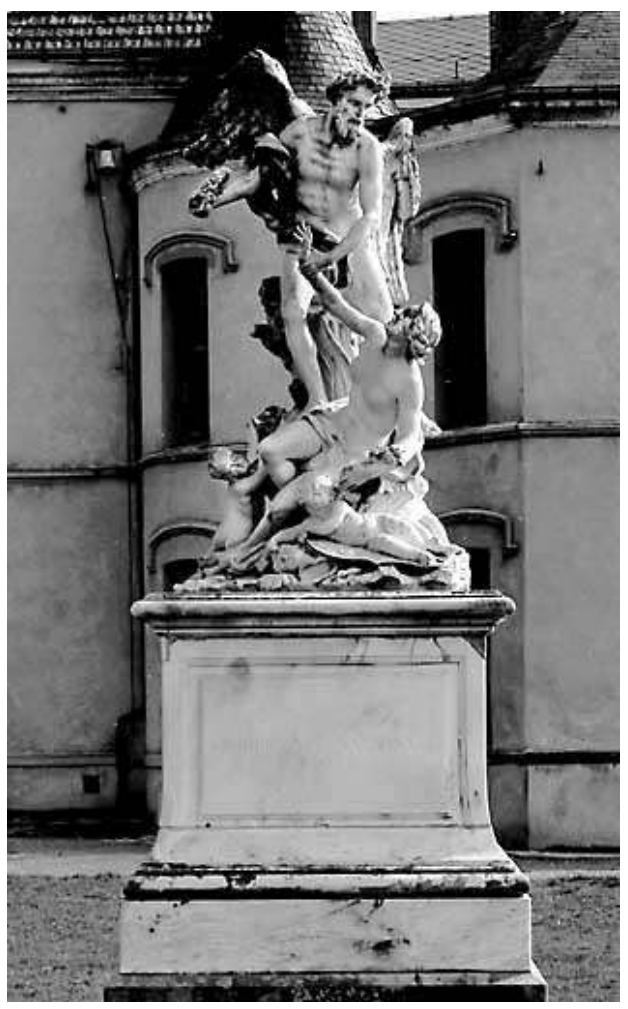

Le temps

Phot. Inv. R. Capponi, () Inventaire général, ADAGP, 1969

11 On peut les admirer aux beaux jours, car elles sont protégées l'hiver dans un étui de paille.

12 Malgré l'assertion quelque peu rapide de Du Parquier, il s'agit en effet d'œuvres destinées à un usage spécifique et dont la qualité répond à cet usage. Elles se déploient en effet dans l'espace et sont parfaitement séduisantes de quelque point de vue où on se place. Le groupe sculpté est la plus élaboré. Taillé dans deux blocs de marbre blanc, il mesure 2,10 $\mathrm{m}$ de haut. 
Figure 2

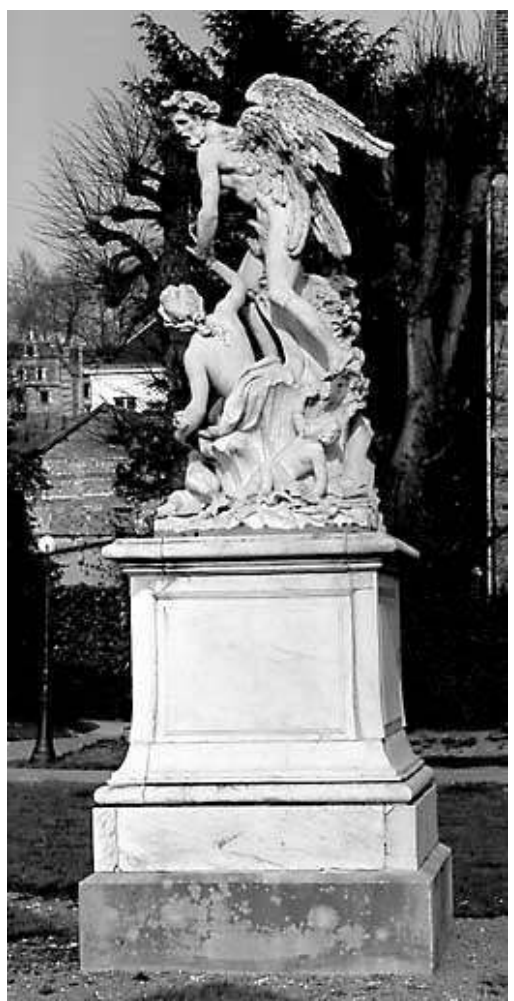

Le temps

Phot. Inv. R. Capponi, (C) Inventaire général, ADAGP, 1969

Le Temps, robuste personnage nu est saisi en plein mouvement, ses ailes encore déployées et son attitude en déséquilibre. Il saisit par le bras un éphèbe nu à demi couché qu'il «relève». Dans son autre main, il tient une couronne de laurier. Les deux personnages s'extraient d'un « fond » stylisé à la fois minéral et végétal, où s'accrochent à l'arrière deux amours nus qui semblent vouloir prêter main forte au Temps, au milieu d'instruments symbolisant l'architecture (compas, règle et équerre), la sculpture (buste et boulier), et la peinture (palette et pinceaux). L'animation et le mouvement des diverses figures, saisies en pleine action, en font un composition pleine de vie. 
Figure 3

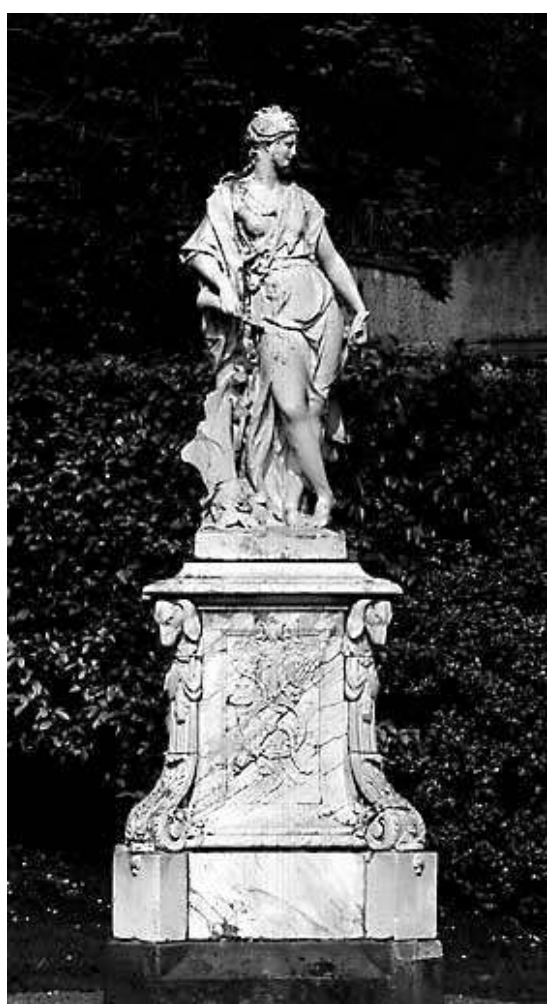

Diane

Phot. Inv. R. Capponi, (C) Inventaire général, ADAGP, 1969

La statue de Diane mesure 1,66 m de haut en un seul bloc de marbre blanc. Son attitude est plus statique, elle dégage d'une main négligente ses jambe nues croisées du voile qui l'enveloppe. Son autre bras s'appuie sur un motif végétal, et elle offre son profil à qui la regarde de face. Le piédestal, orné de quatre bas-reliefs constitue une œuvre à part entière, consacré à l'art cynégétique (carquois, flèches, cor de chasse, poignard, lièvre et perdrix). Aux quatre angles, des têtes de chien somment un motif en volute et sur les quatre faces des trophées d'armes de chasse retenus par des rubans présentent des arcs, carquois, cor ou corne de chasse, pattes de cerf ou hure de sanglier. Il s'agit d'une Diane chasseresse dont la place dans un parc et tout à fait appropriée. Mais dépassant l'intérêt intrinsèque de ces sculptures, leur valeur vient de leur histoire, de cette volonté de " récupération révolutionnaire ", un processus d'appropriation des œuvres d'art de la « classe dominante » par les « exploités », plutôt que leur destruction qui serait une perte pour tous, selon un discours déjà souligné par Jean-Marie Pérouse de Montclos à propos des précurseurs de l'Inventaire général des Monuments et Richesses artistiques de la France $^{21}$ : les monuments révèlent la « génie » d'une nation et font partie du « patrimoine national ». Le Musée des Monuments français créé par Alexandre Lenoir, le « Museum» est une autre manifestation de cette entreprise. 


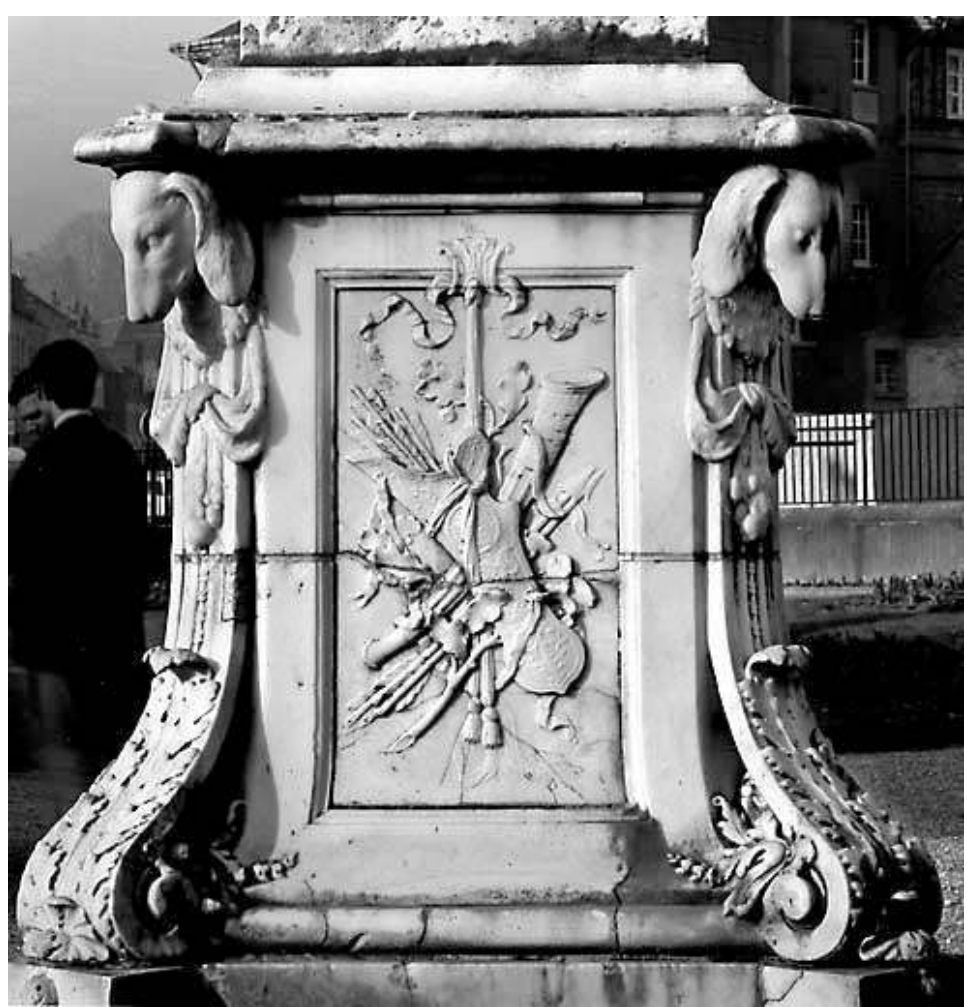

Piédestal de la statue de Diane

Phot. Inv. R. Capponi, () Inventaire général, ADAGP, 1969

Collen-Castaigne, dans son essai sur Bolbec ${ }^{22}$ rapporte le poème composé à l'occasion de l'érection du groupe de Marly qui explicite bien la signification donnée à l'époque à cette action:

«Si le Temps, en passant, donne la main aux Arts,

Il s'envole et revient plus tard pour les combattre.

Il n'est point de donjons, il n'est point de remparts

Que les coups de sa faux n'aient fini par abattre...

A ce monde sans fin, dans plus de dix mille ans,

Ce vieillard fugitif procurera peut-être

Des savants distingués qui prouveront aux gens

Que la saine raison vient à l'instant de naître !».

Tout est en effet à retenir dans cette affaire exemplaire. En premier lieu, l'idée que les dépôts où ont été rassemblées les œuvres d'art sont à la disposition des citoyens, et peuvent servir de "réservoir» d'œuvres destinés à l'édification de la collectivité: Jacques-Dominique Ruffin réclame avec autant d'ingénuité que de naturel ce qui conviendra le mieux pour la décoration de sa ville. En second lieu, cette «munificence nationale » à Bolbec, qui n'est pas la seule, d'autres villes en ayant évidemment bénéficié, est le prélude de la politique continue de « dépots de l'Etat » destinés à enrichir les villes de province d'œuvres soit originales, comme à Bolbec, soit de copies œuvres célèbres proposées pour l'éducation des citoyens. C'est bien ainsi que sont appréhendées à Bolbec ces œuvres qui peuvent à première vue surprendre dans une ville qui n'a rien de particulièrement "artistique ». Dans les années 1960, le directeur du département de sculpture du musée du Louvre qui cherchait à rassembler les vestiges de la statuaire des 
jardins de Marly a proposé à la ville d'échanger ce dépôt de l'Etat contre un dépôt d'œuvres contemporaines du Fonds national d'Art contemporain ${ }^{23}$, mais le conseil municipal a repoussé l'offre à l'unanimité, avec une pointe de réprobation ${ }^{24}$.

17 Notices existantes dans les bases de données nationales : Sculpteurs Jean Poulier et Jean Hardy

BIBLIOGRAPHIE

\section{Pour en savoir plus sur les sculptures du jardin de Marly :}

\section{A la bibliothèque nationale (Tolbiac) : http://catalogue.bnf.fr}

Rosaco, Betsy. The sculptures of the Château of Marly during the Riegn of Louis XIV. New York; London: Garland, 1986. XXXV-637 p. -pl.; ill.; 34 cm. (8-LJ9-8362).

\section{A la BPI (Beaubourg) : http://sbib.ck.bpi.fr}

Mabille, Géard. Vues des jardins de Marly : le roi jardinier. Paris : A. de Gourcuff, 1998. 236 p. : ill. ; $31 \mathrm{~cm}$ (721.8(44)MAB).

\section{A la bibliothèque d'Art et d'Archéologie (Doucet) : http:// www.sudoc.abes.fr}

Nouvelle description des châteaux et parcs de Versailles et de Marly, contenant une explication historique de toutes les peintures, tableaux, statues, vases... / par M. Piganiol de la Force, sixième édition. Paris : Vve Delaulne, 1730. 2 vol. : ill., plans ; $18 \mathrm{~cm}$ (NA7736.V5)

Magne, Emile. Le château de Marly, d'après des docuemnts inédits. Paris : Calmann-Lévy, 1934. 244 p., plans ; $19 \mathrm{~cm}$ (DC801.M3M3).

\section{Articles de revues (Catalogue collectif/Réseau d'histoire de l'art) : http://www.kubikat.org}

Allain-Bernard, Geneviève. Quelques nouvelles identifications de sculptures provenant du parc de Marly. In Bulletin de la Société de l'histoire de l'art français, 1996 (1997), p. 65-77.

Bentz, Bruno. Marly, jardin des eaux. In Histoire de l'art, 12/1990, p. 27-36. 


\section{NOTES}

1. Jacques-Dominique Ruffin (1771-1811), capitaine de la compagnie des volontaires de Bolbec, est nommé commandant du 7e bataillon de la Seine-Inférieure le 20 septembre 1792. Il s'illustre dans les batailles de la Révolution puis de l'Empire notamment à Friedland en 1807, ce qui lui vaut le titre de comte de l'Empire et le grade de général de division. Il s'illustre pendant la campagne d'Espagne et, fait prisonnier devant Cadix, il meurt pendant son transfert en Angleterre des suites de ses blessures le 15 mai 1811 et reçoit de l'armée anglaise les honneurs militaires.

2. Plans de la ville de Bolbec, dressés à l'occasion de la grande route de Rouen au Havre, liste des propriétaires sur le terrain desquels elle doit passer, 1765 (A.D. 76 C 1103).

3. 13 novembre 1583, décharge d'imposition en faveur du bourg de Bolbec, en considération de la pauvreté ou auroient été réduictz les habitans du bourg de Bollebec par accident du feu...ayant brullé jusques à VIIIc (800) maisons et autres sortes de logis, mesmement le clocher dudict lieu (A.D. 76 C 1269).

4. Requêtes et ordonnances du bureau des Finances relatives aux alignements donnés à l'effet de bâtir sur la nouvelle route de Rouen à Bolbec, plan colorié, 1774-1775 (A.D. 76 C 2369)

5. Dès 1785, François-Henri d'Harcourt seigneur haut-justicier de Lillebonne, propriétaire de la place du Marché et des marchés de Bolbec, avec siège de la justice à l'étage de la halle (la cohue ou prétoire) cède à la ville un terrain pour y construire un hôtel de ville à condition d'indemniser les locataires des logettes. Bien national de 2e origine à la Révolution, la place devient propriété de la nation qui la remet à la ville. La première pierre de l'hôtel de ville est posée en 1791 .

6. Projets, plans, coupes et élévations, vers 1765-1770, par Pierre Patte (A.M. Bolbec M2)

7. Délibérations du conseil municipal, 9 frimaire an II (A.M. Bolbec).

8. Service des eaux, 8 floréal an II. Devis des ouvrages à exécuter en terrasse, maçonnerie, charpente, conduite d'eau pour l'établissement de trois fontaines publiques (A.M. Bolbec N3 1794 $->)$.

9. 28 ventôse an III : arrêté concernant l'établissement de 3 fontaines publiques (A.M. Bolbec. Notes Brianchon)

10. Lettre de Jacques-Dominique Ruffin à la Convention nationale, 22 messidor en II ( A.M. Bolbec. Ibid.).

11. Château de Marly-le-Roi. Extraction des objets de l'art du parc du château, 11 frimaire an II (A.D. Seine-et-Oise. C W $20 \mathrm{n}^{\circ}$ 4776).

12. Certificat de la Commission exécutive de l'Instruction publique. 16 frimaire an III (A.M. Bolbec. Notes Brianchon).

13. Lettre du Comité d'Instruction publique (ibid.).

14. Délibération du comité de la commune de Bolbec, 15 brumaire an IV (A.M. Bolbec).

15. Le sculpteur Jean Poultier (1653-1719), reçu à l'Académie en 1684 pour la Vierge et saint Jean destinés à l'entrée du chœur de Saint-Nicolas du Chardonnet a exécuté en 1702 quatre statues pour les jardins de Versailles, dont la « femme assise pleurant et tenant un miroir », classée M.H. en 1905.

16. Le sculpteur Jean Hardy (1653-1737) a été reçu à l'Académie en 1688. Le Musée de Chantilly conserve 6 statues de nymphes et le Musée du Louvre deux groupes.

17. Furcy-Raynaud. Communication à la Société de l'Histoire de l'Art français, le 14 mai 1909 (A.M. Bolbec. Dossier Monuments historiques).

18. La fontaine du Temps, dessin par Nicétas Périaux, litho. par Dumas, 1839. L'ancienne fontaine de la place du Marché, carte postale D. Laisné à Bolbec, début 20e siècle. 
19. L'ancienne fontaine de la place Diane, carte postale $\mathrm{D}$. Laisné à Bolbec, début $\mathrm{XX}^{\mathrm{e}}$ siècle. Ces deux cartes postales sont des reproductions de photographies datant d'avant 1887, date du déménagement des statues.

20. Raymond Bernard. 1887, une grande année pour Bolbec ou la vie locale avec un siècle de distance, recueil factice d'articles parus dans la presse bolbécaise en 1887. Bolbec, 1987. Il s'agit du sculpteur rouennais Bonnet fils, qui a travaillé à la restauration de l'église paroissiale SaintPatrice avec l'architecte Louis Sauvageot.

21. Ministère de la Culture et de la Communication. L'Inventaire général des Monuments et des Richesses artistiques de la France. Paris, 1978.

22. Collen-Castaigne. Essai historique et statistique sur la ville de Bolbec. Rouen: N. Periaux, 1839.

23. Le Fonds national d'Art contemporain (F.N.A.C.) a en effet repris la politique de dépôts de l'Etat mis en œuvre avant la seconde guerre par le Ministère de l'Education nationale, section Beaux-Arts.

24. Délibérations du Conseil municipal de Bolbec (A.M. Bolbec).

\section{INDEX}

Mots-clés : inventaire général, en ligne, journal, revue électronique, revue numérique, périodique, patrimoine, histoire de l'art, France, Haute-Normandie, Paris, Bolbec, Marly, Lenoir Alexandre, sculpture, $18 \mathrm{e}$ siècle, $19 \mathrm{e}$ siècle

Keywords : on line, electronic journal, ejournal, heritage, history of art, Normandy, XVIIIth century, XIXth century

\section{AUTEUR}

\section{CLAIRE ETIENNE}

Conservateur du patrimoine, Sous-direction des études, de la documentation et de l'Inventaire Hôtel de Vigny 10, rue du Parc Royal 75003 Paris. claire.etienne@culture.gouv.fr 